\title{
Multipath Estimation Technique for Wideband mm-Wave Backscattering Channels
}

\author{
Francesco Guidi, Antonio Clemente, Raffaele D'Errico \\ CEA, LETI, MINATEC Campus, 38054 Grenoble, France. \\ Univ. Grenoble-Alpes, 38000 Grenoble, France \\ e-mail: \{francesco.guidi, antonio.clemente, raffaele.derrico\}@cea.fr
}

\begin{abstract}
In this paper we describe a method to discriminate multipath components in joint angular and temporal domain by exploiting the a-priori knowledge of the antennas characteristics into an ad-hoc algorithm for wideband backscattering channels. By means of two indoor measurement campaigns performed using millimeter-waves massive arrays in a corridor and in an office room, we show the feasibility of the proposed approach and we compared its performance to those of methods already available in the state of the art.
\end{abstract}

Index Terms-Multipath Detection, Massive antenna array, personal radar.

\section{INTRODUCTION}

Next fifth generation (5G) wireless mobile networks is still at its infancy, as its explosion and practical adoption are expected in the next decade [1]. Among the new disruptive players for $5 \mathrm{G}$ wireless mobile networks, it is foreseen to have a wide-spread adoption of millimeter-wave ( $\mathrm{mmW}$ ) and massive arrays technologies. Consequently, by taking advantage of $\mathrm{mmW}$ which gives the possibility to pack a huge number of antennas into a small area, next generation of mobile devices could be able to scan the surrounding environment thanks to the beamsteering capabilities enabled by the integrated massive arrays [2], [3]. From this perspective, personal radars exploiting the joint use of these technologies represent an interesting solution for environment mapping [4], [5]. Notably, the performance of such system depends on: (i) the ability of collecting the backscattered responses from different steering directions; (ii) the exploitation of such measurements as an input for the mapping algorithm. As a consequence, it becomes important to properly characterize the indoor backscattering $\mathrm{mmW}$ channel. To this purpose, the first step is to properly detect the multipath components (MPCs) in a 2D scenario, where joint angular and temporal information should be accounted for. At the best of authors' knowledge, an extensive literature is available on CLEANlike approaches, their extensions, or on different techniques [6]-[12], but all these methods to retrieve the MPCs, related to the one-way channel, do not usually account for the need to reconstruct the environment. In [11] and [12], extended versions of the CLEAN algorithm accounting for both space and time dimension are described. More specifically, in [11] a Sensor-CLEAN algorithm is proposed, which exploits arrays of measurements to reconstruct the beam direction and time response by means of delay-and-sum beamforming. In such approach, requiring the construction of the beamforming matrix, data from different sensors (antennas) is gathered, and the output of the CLEAN provides an estimate of the timeof-arrival (TOA), angle-of-arrival (AOA) and pulse shape. In [12] instead, authors reasonably assume to have point sources blurred due to noise and point-spread-functions (i.e. impulse responses). Thus, once a point-spread-function is estimated, it is exploited to iteratively clean the measured data along the angular and time dimension. A misalignment in the use of this function can cause further artifacts, thus requiring detection schemes with constant false alarm rates, or visual inspection to correct the unwanted gathered components. In [13], an approach accounting for joint temporal and angular information of the collected MPCs in a different manner is proposed.

In this paper, inspired by these methods, we propose an extended version of the CLEAN algorithm to detect MPCs by exploiting the joint delay and angular information in wideband backscattering channels with antenna pattern embedded. More specifically, the a-priori information of the high-directive antenna patterns used for measurements is exploited to reduce as much as possible the effect of arrays side-lobes. The output of the proposed algorithm is compared to that of a more traditional CLEAN-like approach, and of the algorithm described in [13], with the aim to put in evidence the benefits carried out by the new approach. Performance is shown by exploiting measurements collected in the V-band adopting transmitarrays (TAs) in two typical office environments at CEA-Grenoble premises, that is an office room and a corridor. In particular, since the exploited measurements are for applications which aim to reconstruct the environment (i.e. for personal radars), through the proposed approach we enable the possibility to reproduce the environment contour.

The rest of the paper is organized as follows. Sec. II reports the description of the proposed method, as well as of the two considered approaches in the state of the art for the comparison. In Sec. III we briefly describe the performed measurement campaign, while in Sec. IV results are reported. Finally, Sec. V draws the conclusions.

\section{Multipath Components Detection}

In our scenario, we assume to have a user equipped with a high directional $\mathrm{mmW}$ antenna which moves in an unknwon 
environment. For each position, thanks to the beamsteering capabilities of its device, the user is able to steer the antenna beam towards different directions, and to collect the backscatter response and thus to reconstruct the surrounding environment. To this purpose, it is important to correctly discriminate the MPCs of such a backscattering channel. Thus, we now first describe two possible approaches reported in the state of the art, as well as the proposed method based on the a-priori knowledge of the antenna pattern.

\section{A. Algorithm 1: CLEAN-like Approach}

We now consider a CLEAN-like approach which follows the following steps.

1) For each radar position, generate a temporal-angular map containing the power delay profiles (PDPs) associated to each steering direction for the different delays;

2) Find the maximum value $P_{\max }$ in the PDPs matrix, and store the corresponding delay, angle and amplitude.

3) Considering $P_{\max }$ as a center point along the delay-axis, set to zero the samples along $\Delta \tau$. A new cleaned version of the map is obtained, where the local maximum is no more present.

4) If at least a value of the new version of the map is above a pre-defined threshold (e.g. a noise-based one), go to step 2. Otherwise continue to step 5;

5) The recorded elements with a total power above a threshold to be set, give the multipath components with their relative angles and delays.

As it is possible to observe, this simple approach does not account for any angular information of the pattern. Thus, it is quite immediate to conclude that such a method is extremely sensitive to the presence of artifacts due to side-lobes as will be also shown in the results section.

\section{B. Algorithm 2: 2D Approach}

We now consider an approach which can be easily adapted to our scenario and that exploits both the angular and temporal information [13]. In particular, from the knowledge of all the measured angular channel responses and their respective PDPs, a marginal PDP is derived by averaging the PDP for all the steering directions. Successively the obtained marginal PDP, which only depends on the delay, is divided into time bins. For each bin, the local maximum is first collected and then compared with a time-dependent threshold, as defined in [13], which is related to the average power level in the bin. If a value overcomes the threshold, such power value and time instant are gathered, as well as the azimuth angle (i.e. steering direction) for which the maximum is achieved at that given time instant by considering all the angular PDPs. In such a manner, for each MPC three parameters are stored.

One could note that for each value crossing the threshold, only one azimuth angle is accounted for. This means that in symmetric environments, where two strong components are collected from opposite steering directions and arrive at about the same time, there is the risk that a useful MPC is discarded.
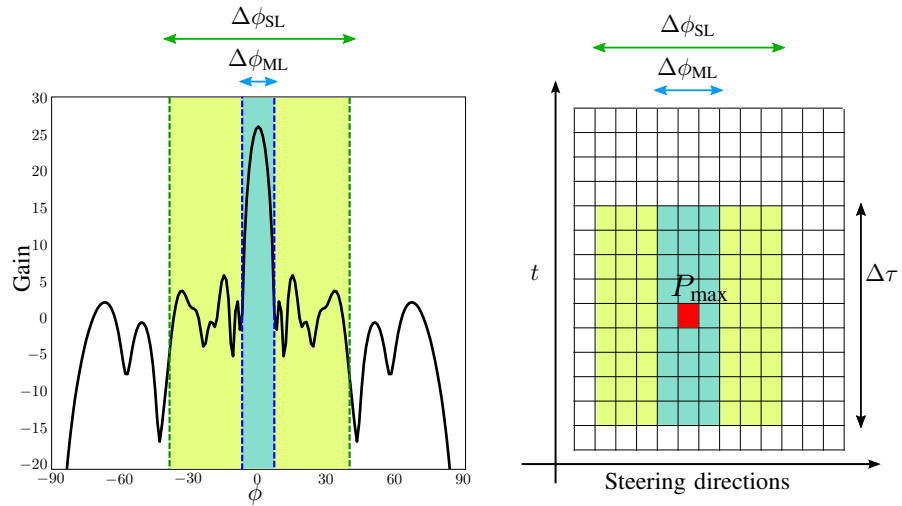

Fig. 1. Array pattern (left) and PDP matrix (right) for multipath components identification in the proposed algorithm 3 .

\section{Algorithm 3: Proposed Approach}

In order to overcome part of the aforementioned limitations, and inspired by the necessity to account for the antenna characteristics in the MPCs search, we describe here a variant of the CLEAN approach, which lets to discriminate angular and time components from the knowledge of the antenna pattern (we suppose here high directive antennas). This algorithm starts from the definition of the PDP matrix for all the steering directions, and of a delay and two spatial windows. In particular, let $\Delta \phi_{\mathrm{ML}}$ and $\Delta \phi_{\mathrm{SL}}$ to be set in accordance to the array pattern, as reported in the example of Fig. 1-left. $\Delta \phi_{\mathrm{ML}}$ accounts for the main lobe, whereas $\Delta \phi_{\mathrm{SL}}$ considers the angles where the side lobe level (SLL), defined as SLL $=G_{\mathrm{sl}} / G_{\max }$, is higher than a pre-determined value. Then, we indicate with $\Delta \tau$ the temporal window representing the estimated multipath component duration. Once the space and time windows are defined, the two-dimensional version of the CLEAN algorithm follows the hereafter enumerated steps.

1) For each radar position, generate a temporal-angular matrix containing the PDPs associated to all the steering directions;

2) Find the local maximum value $P_{\max }$ in the PDPs map, and store its delay, angle and value. In Fig. 1-right this value is represented as a red-colored entry of the spacetime matrix;

3) Considering $P_{\max }$ as a center point, set to zero the samples in the area between $\Delta \phi_{\mathrm{ML}}$ and $\Delta \tau$ according to Fig. 1-right. A new cleaned version of the map is obtained, where the local maximum is no more present.

4) Find if in $\Delta \phi_{\mathrm{SL}}$, there is any component with power $P_{\mathrm{SL}}>\xi=P_{\max } / \zeta$, with $\zeta=k \cdot\left(G_{\max } / G_{\mathrm{sl}}\right)^{2}$ accounting twice the side-lobes effects as the antenna gain enters in the transmitting and receiving mode due to the backscattering nature of the communication, and with $G_{\text {sl }}$ and $G_{\max }$ being the side-lobe and maximum gain, respectively. $k$ is a constant which lets to better balance the threshold. If there is no component overcoming the threshold, set to zero the samples in the area comprised by $\Delta \phi_{\mathrm{SL}}$ and $\Delta \tau$ by taking the maximum peak as a 

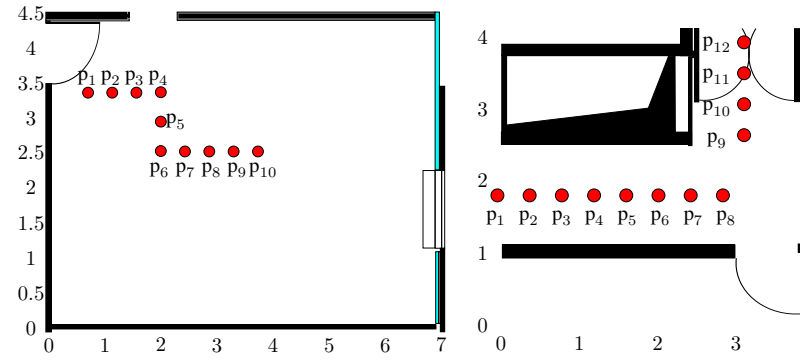

Fig. 2. Plan of the indoor office environment.

center. This operation permits to check if the samples around the considered local maximum are artifacts due to side-lobes and hence they should be discarded or not. In fact, components whose correspondent power is above the threshold could arrive from other steering directions and they should be collected;

5) If at least a value of the new version of the map is above a pre-defined threshold (e.g. a noise-based one), go to step 2. Otherwise continue to step 6;

6) The recorded elements with a total power above a threshold to be set give the multipath components with their relative angles and delays.

In the following, after reporting the measurement campaign, the algorithms performance is shown.

\section{Channel Measurement CAMPAigns}

We now describe two measurement campaigns performed in order to assess a characterization of the backscattering channel model, which will be here exploited to assess the performance of the proposed method. Measurements were performed in an office room and a corridor, as shown in Fig. 2. To this purpose, $2 \mathrm{mmW}$ converters in $50 \mathrm{GHz}-75 \mathrm{GHz}$ were connected from one side to a vector network analyzer and, from the other side, to 2 linearly polarized TAs with $20 \times 20$ elements each, 1 bit of phase quantization, and a focal distance $F / D=0.5$ [14]. The entire set-up was positioned over a $X$-Y-Azimuth positioner to let possible the mechanical rotation of the structure and thus emulating a steering procedure. In particular, the 2 TAs were placed in a quasi monostatic configuration and spaced apart of $0.16 \mathrm{~m} \mathrm{[5],} \mathrm{[15].} \mathrm{As} \mathrm{reported} \mathrm{in} \mathrm{Fig.} \mathrm{2,} \mathrm{in} \mathrm{order}$ to emulate the personal radar scenario of [4], measurements were collected in 10 and 12 different positions spaced apart of $0.405 \mathrm{~m}$. For each point, steering operations were performed by mechanically rotating the $X$-Y-Azimuth positioner from $-90^{\circ}$ to $90^{\circ}$ with a step of $5^{\circ}$ (in accordance to the half power beamwidth (HPBW) of the TA used), in the $57.5-63.5 \mathrm{GHz}$ bandwidth with a step of $5 \mathrm{MHz}$. Successively, we reported the measured $\mathbf{S}_{21}(f)$ in time-domain through the inverse Fourier transform, and we performed time gating to eliminate the coupling contribution due to the two arrays proximity.

\section{A. Massive Array Characteristics}

We now give some insights concerning the TA characteristics used for measurements. The aim is then to exploit
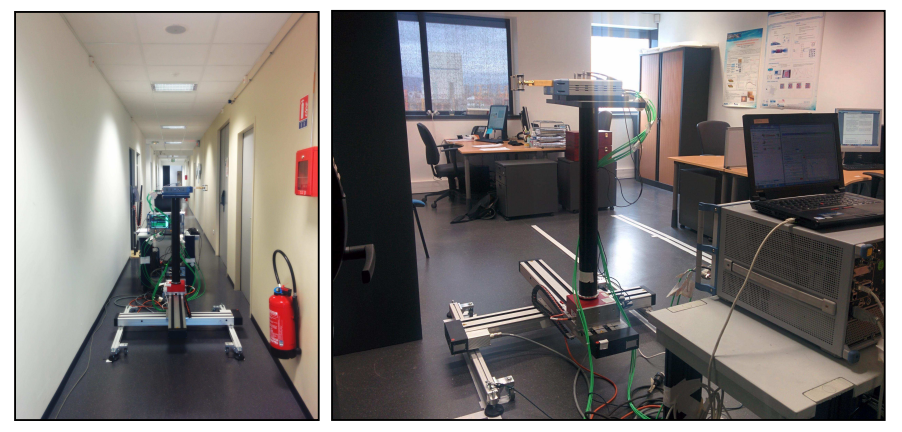

Fig. 3. Photos of measurement campaigns in corridor.

such information into the algorithm described in Sec. II-C. The considered multi-antenna systems are composed of a focal source illuminating a planar array constituted of blocks called unit-cells. To achieve high directivity for personal radar applications, it has been shown that TAs with 400 elements are a good choice [5], [14], [16]. Consequently, we considered $20 \times 20$ unit-cells each with size of $2.5 \times 2.5 \mathrm{~mm}^{2}$ (i.e. the spacing between antennas is set to $\lambda_{0} / 2=5 \mathrm{~mm}$ where $\lambda_{0}$ is the wavelength at $f_{0}=60 \mathrm{GHz}$ ). The considered TA presents 1-bit of phase compensation corresponding to two possible phase values $\left(0^{\circ}\right.$ and $\left.180^{\circ}\right)$.

Finally, the focal source is a linearly-polarized pyramidal horn antenna with a gain equal to $10.2 \mathrm{dBi}$ at $60 \mathrm{GHz}$ and a full HPBW of $72^{\circ} \times 57^{\circ}$. Results report a maximum gain of $23.3 \mathrm{dBi}$, a directivity, spill-over loss and power efficiency equal to $26 \mathrm{dBi}, 2.24 \mathrm{~dB}$, and $53.6 \%$, respectively. Since we are also interested in the side-lobe region, from the gain characteristics we have that the SLL can be higher than $-20 \mathrm{~dB}$ in the region $\pm 40^{\circ}$ centered in the maximum gain direction, that is $\mathrm{SLL}=G_{\mathrm{sl}}-G_{\max }>-20 \mathrm{~dB}$. As previously mentioned, such array characteristics are matching with the personal radar need to have extremely high directive antennas in order to scan the environment and made the antenna compact and suitable for testing personal radar applications. For more details concerning the TAs, we refer the reader to [14].

\section{RESULTS}

We now compare the performance of the previously considered algorithms. In particular, by visual inspection, we show how such known methods fail in reconstructing the environment perimeter. As previously explained, all the three methods start from the generation of the joint angular and temporal matrix for each position, given by putting together measured data considering all the steering directions.

A temporal window of $\Delta \tau \approx 1.5 \mathrm{~ns}$ has been considered for the three approaches, which has been estimated through the TAs coupling. More specifically, for the algorithm 3, according to Sec. II-C and to the TA described in Sec. III, we set $\Delta \phi_{\mathrm{ML}}= \pm 5^{\circ}$ and $\Delta \phi_{\mathrm{SL}}= \pm 40^{\circ}$ both centered in the maximum gain. Fig. 1-left shows how the spatial windows are accounted for in the gain pattern. In particular, the value of $\pm 5^{\circ}$, which means to account for a steering direction on the 


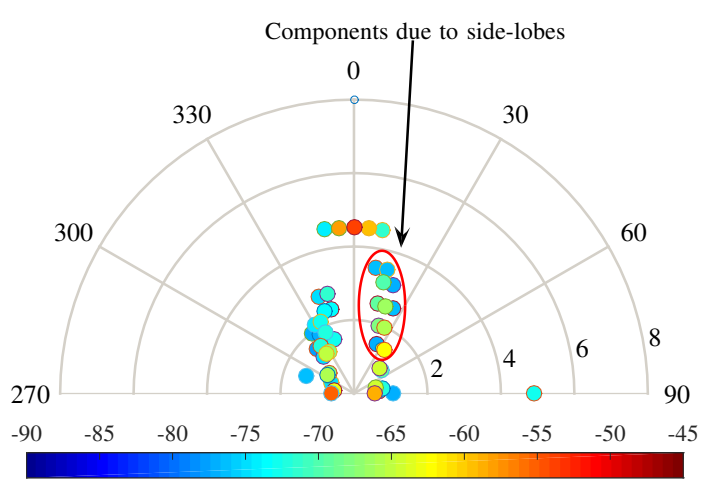

(a) Algorithm 1

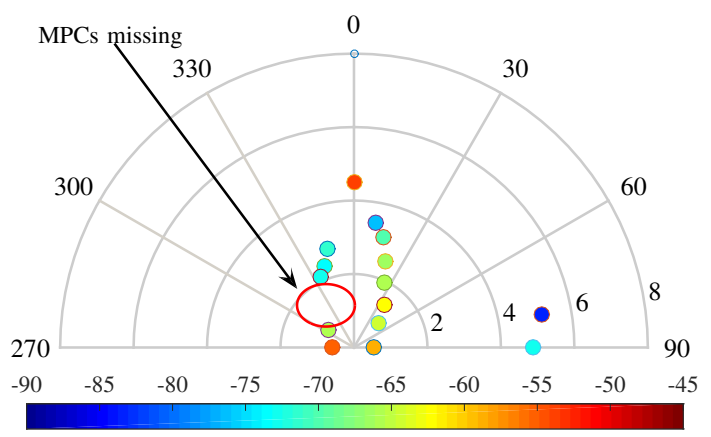

(b) Algorithm 2

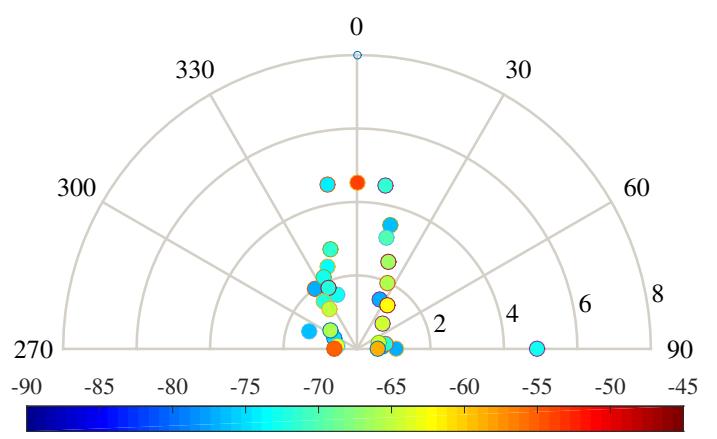

(c) Algorithm 3

Fig. 4. Identified MPCs for the corridor environment with the considered algorithms.

left and on the right from the maximum, has been estimated by considering the $-10 \mathrm{~dB}$ main lobe.

Figs. 4 and 5 show the comparison of the obtained maps for the corridor and the room, respectively. By accounting for algorithm 1 (Fig. 4-top and Fig. 5-top), it is important to notice that for each retrieved component, we experience the presence of several contributions which can be intuitively associated to those of side-lobes. Consequently, as also reported in Table I, the number of MPCs is overestimated and need to be corrected.

When instead the algorithm 2 is considered (Fig. 4-middle

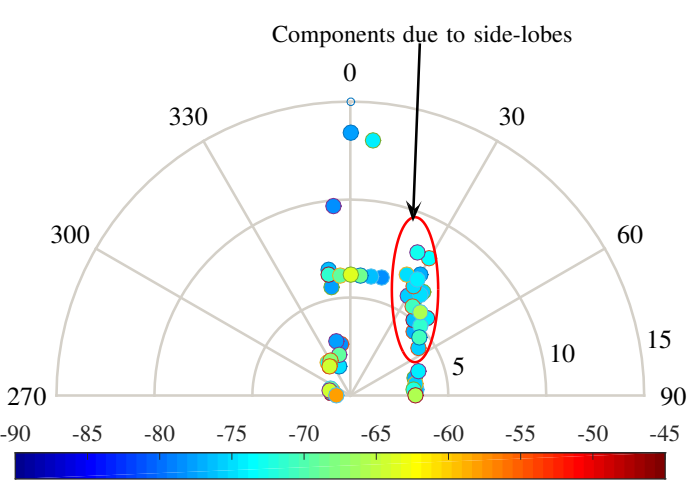

(a) Algorithm 1

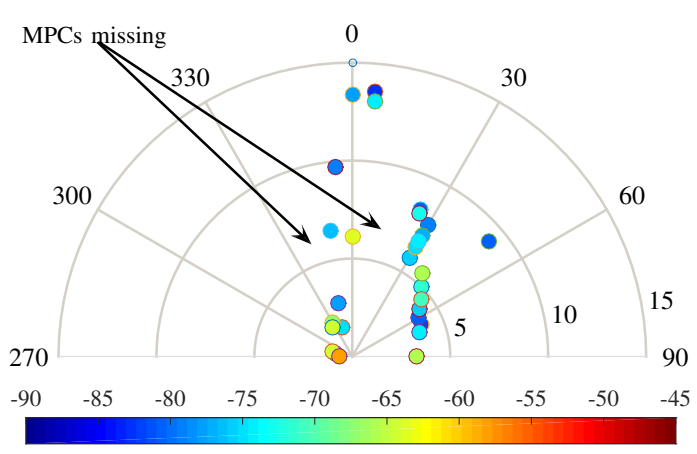

(b) Algorithm 2

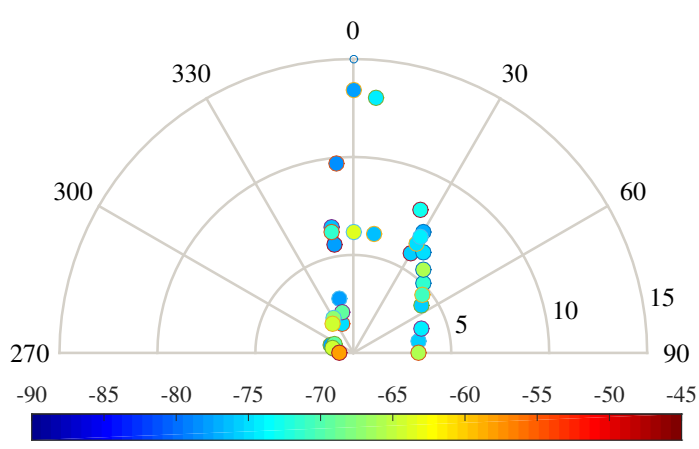

(c) Algorithm 3

Fig. 5. Identified MPCs for the office room environment with the considered algorithms.

and Fig. 5-middle), there is another main limitation. In fact, if there is environment symmetry with respect to the radar position, useful components could be discarded. As also reported in the two figures, the detection of specular components is often missed each time two components fall in the same time window. Contrarily to the adoption of the algorithm 1, the number of paths risks to be underestimated, especially for what the corridor (i.e. a more symmetric environment) is concerned (see Table II).

Thus, we suffer from two competing effects: from one side 
TABLE I

ALGORITHM 1 (CLEAN-LIKE APPROACH): NUMBER OF PATHS PER POSITION.

\begin{tabular}{|c|c|c|c|c|c|c|c|c|c|c|c|c|}
\hline Position & $p_{1}$ & $p_{2}$ & $p_{3}$ & $p_{4}$ & $p_{5}$ & $p_{6}$ & $p_{7}$ & $p_{8}$ & $p_{9}$ & $p_{10}$ & $p_{11}$ & $p_{12}$ \\
\hline Corridor & 55 & 57 & 66 & 45 & 47 & 33 & 23 & 26 & 42 & 40 & 38 & 52 \\
Room & 61 & 48 & 53 & 74 & 49 & 49 & 71 & 75 & 61 & 48 & - & - \\
\hline
\end{tabular}

TABLE II

ALGORITHM 2 [13]: NUMBER OF PATHS PER POSITION.

\begin{tabular}{|c|c|c|c|c|c|c|c|c|c|c|c|c|}
\hline Position & $p_{1}$ & $p_{2}$ & $p_{3}$ & $p_{4}$ & $p_{5}$ & $p_{6}$ & $p_{7}$ & $p_{8}$ & $p_{9}$ & $p_{10}$ & $p_{11}$ & $p_{12}$ \\
\hline Corridor & 16 & 11 & 15 & 12 & 17 & 13 & 18 & 16 & 16 & 16 & 17 & 13 \\
Room & 31 & 26 & 21 & 21 & 16 & 17 & 25 & 29 & 23 & 21 & - & - \\
\hline
\end{tabular}

TABLE III

ALGORITHM 3 (PROPOSED APPROACH): NUMBER OF PATHS PER POSITION.

\begin{tabular}{|c|c|c|c|c|c|c|c|c|c|c|c|c|}
\hline Position & $p_{1}$ & $p_{2}$ & $p_{3}$ & $p_{4}$ & $p_{5}$ & $p_{6}$ & $p_{7}$ & $p_{8}$ & $p_{9}$ & $p_{10}$ & $p_{11}$ & $p_{12}$ \\
\hline Corridor & 30 & 26 & 31 & 21 & 23 & 13 & 11 & 13 & 22 & 20 & 20 & 31 \\
Room & 34 & 25 & 28 & 38 & 23 & 23 & 37 & 36 & 31 & 22 & - & - \\
\hline
\end{tabular}

we risk to avoid symmetric components of the environment if we use algorithm 2, and from the other we might account for components due to side-lobes if we consider only a time window as in algorithm 1 . A compromise is required in order to preserve a good balance between the number of components and undesired effects. As also previously underlined, the main goal is to collect MPCs which let to characterize the environment contour. By looking at Fig. 4-bottom and Fig. 5bottom, it is possible to observe the benefits carried out by the proposed method for the previously considered four positions in the corridor and in the room, respectively. As it is possible to observe, the selected components follow the environment contour in line with the objective to find a proper channel modeling for environment mapping. Notably, the environment contour is much more defined with respect to the previous cases, and spurious components are avoided.

\section{CONCLUSION}

In this paper, an extended version of the CLEAN algorithm is described in order to discriminate the useful MPCs. Based on mmW backscattering measurements collected from different radar positions and steering directions, through the proposed CLEAN-like mechanism we enabled the possibility to determine the joint angular and delay information of the paths, thus reconstructing the contour of the environments. The obtained paths can be post-processed to obtain a $\mathrm{mmW}$ backscattering channel model for personal radars applications.

\section{ACKNOWLEDGMENTS}

This research was supported in part by the IF-EF MarieCurie project MAPS (Grant 659067). Authors would like to thank Anna Guerra for her work in the measurement campaign and for the fruitful discussions.

\section{REFERENCES}

[1] http://www.huawei.com/5gwhitepaper/.
[2] W. H. Chin, Z. Fan, and R. Haines, "Emerging technologies and research challenges for $5 \mathrm{G}$ wireless networks," IEEE Wireless Commun., vol. 21, no. 2, pp. 106-112, April 2014.

[3] T. Rappaport et al., in Millimeter Wave Wireless Communications. Prentice Hall, 2014.

[4] F. Guidi, A. Guerra, and D. Dardari, "Personal mobile radars with millimeter-wave massive arrays for indoor mapping," IEEE Trans. Mobile Comput., 2015.

[5] A. Guerra et al., "Millimeter-wave backscattering measurements with transmitarrays for personal radar applications," in Proc. IEEE Global Conf. on Commun. (GLOBECOM), LION Workshop, 2015.

[6] P. F. Smulders, "Statistical characterization of $60-\mathrm{GHz}$ indoor radio channels," IEEE Trans. Antennas Propag, vol. 57, no. 10, pp. 28202829, 2009.

[7] H. Xu, V. Kukshya, and T. Rappaport, "Spatial and temporal characteristics of 60-GHz indoor channels," IEEE J. Sel. Areas Commun., vol. 20, no. 3, pp. 620-630, Apr 2002.

[8] T. Manabe, Y. Miura, and T. Ihara, "Effects of antenna directivity and polarization on indoor multipath propagation characteristics at $60 \mathrm{ghz}$," IEEE J. Sel. Areas Commun., vol. 14, no. 3, pp. 441-448, Apr 1996.

[9] P. Pajusco, N. Malhouroux-Gaffet, and G. El Zein, "Comprehensive characterization of the double directional UWB residential indoor channel," IEEE Trans. Antennas Propag., vol. 63, no. 3, pp. 1129-1139, 2015.

[10] C. Gustafson et al., "On mm-wave multipath clustering and channel modeling," IEEE Trans. Antennas Propag., vol. 62, no. 3, pp. 14451455, 2014.

[11] R.-M. Cramer, R. A. Scholtz, and M. Z. Win, "Evaluation of an ultrawide-band propagation channel," IEEE Trans. Antennas Propag., vol. 50, no. 5, pp. 561-570, 2002.

[12] Q. H. Spencer et al., "Modeling the statistical time and angle of arrival characteristics of an indoor multipath channel," IEEE J. Sel. Areas Commun., vol. 18, no. 3, pp. 347-360, March 2000.

[13] K. Haneda et al., "A statistical spatio-temporal radio channel model for large indoor environments at 60 and $70 \mathrm{GHz}$," IEEE Trans. Antennas Propag., vol. 63, no. 6, pp. 2694-2704, 2015.

[14] H. Kaouach et al., "Wideband low-loss linear and circular polarization transmit-arrays in V-band," IEEE Trans. Antennas Propag., vol. 59, no. 7, pp. 2513-2523, July 2011.

[15] A. Guerra et al., "Delay spread characterization of millimeter-wave in door backscattering channel," in Proc. 10th European Conf. on Antennas and Propag. (EuCAP), April 2016.

[16] A. Clemente et al., "Wideband 400-element electronically reconfigurable transmitarray in X band," IEEE Trans. Antennas Propag., vol. 61, no. 10, pp. 5017-5027, Oct 2013 . 